\title{
Carnets
}

Revue électronique d'études françaises de l'APEF

Deuxième série - 7| 2016

Plurilinguisme et migrations dans la littérature de langue française

\section{Langue-frontière ou langue-franche : L'hétérolinguisme dans la prose afropéenne et cubano-américaine}

Apories et traduction chez Léonora Miano et Cristina García

\section{Ferdulis Zita Odome Angone}

\section{OpenEdition}

\section{Journals}

Édition électronique

URL : http://journals.openedition.org/carnets/1026

DOI : $10.4000 /$ carnets. 1026

ISSN : 1646-7698

Éditeur

APEF

\section{Référence électronique}

Ferdulis Zita Odome Angone, « Langue-frontière ou langue-franche : L'hétérolinguisme dans la prose afropéenne et cubano-américaine », Carnets [En ligne], Deuxième série - 7 | 2016, mis en ligne le 31 mai 2016, consulté le 19 avril 2019. URL : http://journals.openedition.org/carnets/1026 ; DOI : 10.4000/ carnets. 1026

Ce document a été généré automatiquement le 19 avril 2019.

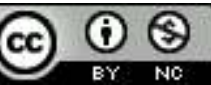

Carnets est mis à disposition selon les termes de la licence Creative Commons - Atribution - Pas d'utilisation commerciale 4.0 International. 


\title{
Langue-frontière ou langue- franche : L'hétérolinguisme dans la prose afropéenne et cubano- américaine
}

\author{
Apories et traduction chez Léonora Miano et Cristina García
}

Ferdulis Zita Odome Angone

I do not want or need to accept the either/or definition of my identity which demands that you choose sides. My identity is far more complex than this. I was born in Havana. I was raised in Texas (...) I returned to Cuba and thus was ostracized from my community. Now I live in Chicago, but also live in Havana, emotionally and professionally (...) I now understand that I do not have to accept categories which split who I am. Instead I must construct new categories, new political and emotional spaces in which my multiple identities can be joined.

(María de los Ángeles Torres, 1994)

À l'ère de l'éloge de l'hybridité, notre réflexion a pour but de faire dialoguer les convergences, les interférences et les différences spécifiques aux écritures diasporiques soumises à notre étude. En effet, baigné par le monde qui l'entoure, le prosateur migrant ou diasporique vit dans l'antre des traductions intersystémiques à travers l'hétérolinguisme autour de lui, un imaginaire où la surconscience babélique des langues foisonne en échos retentissants. Les pratiques langagières rhizomorphiques, inhérentes au chaos-monde ${ }^{1}$, font de ce marqueur de paroles le producteur privilégié d'une poétique de l'étrangeté où le divers, le composite augure d'un engagement dans la langue, un langagement dirait Lise Gauvin (2000). Ainsi, loin de se soumettre à la domination glottopolitique voire glottophagique (Calvet, 1974/2002) de la langue officielle, d'usage claquemurée dans un monolinguisme sourd, les langues minorisées ou minorées ${ }^{3}$, sur/ 
vivant en situation diglossique, résistent avec argutie afin de "contaminer » ou d'« attaquer » la langue plénipotentiaire. Des langues fantômes dont les sonorités nous habitent, nous hantent, coupées de la mémoire du temps. On les entend qui raisonnent en sourdine, ces langues d'autrefois qui tanguent et se fanent sans jamais nous quitter (cf. Fleischer, 2005; Kristeva, 2007). Contrebandiers des langues hautaines, à travers le renouvellement esthétique qu'ils en font, les écrivains hybrides, chacun de leur voix brise ainsi un territoire, efface une frontière, renversant une limite au moyen d'une pollinisation croisée (cf. Chamoiseau, 1997).

2 Notre analyse comparée va se focaliser sur l'hétérolinguisme comme faits et effets de langue, en soulignant les apories observées dans les littératures issues de l'entre-deux. Par le biais de la note comme zone de périphérie narrative, le prosateur migrant y trouve d'une part un palliatif pour suppléer les manquements d'un langage incommensurable qui ne traduit pas toujours à suffisance les apories de son univers hétérotopique. D'autre part, en tant que recours privilégié de l'univers paratextuel, l'écrivain issu des espaces minorisés s'en sert comme stratégie poétique pour déjouer les normes idéologiques d'un canon monolingue, dominant. La note représente donc dans l'intention poétique de ce dernier, une sorte de zone-tampon, une marge dans la marge car notes explicatives, glossaires, accessoires narratifs et adjuvants stylistiques viennent à la rescousse d'un texte où les formes esthétiques se situent déjà à la périphérie d'un centre aux canons souvent hiératiques. Chez Miano la francophone, on étudiera les notions de l'oralité africaine à travers le conte tandis que chez la cubano-américaine d'expression anglaise Cristina García, nous parlerons du spanglish (ou espanglish ou encore espanglés) ${ }^{4}$ avec pour paradigme la santería cubaine. Le propos ici vise à mettre en exergue les littératures minorées au cœur de notre réflexion. Notre approche théorique sera pluridisciplinaire parce qu'un texte hybride, c'est-à-dire hétérogène donc à ancestralités multiples, requiert un regard pluriel pour mieux apprécier la polysémie de l'âme métisse des identitésfrontières.

\section{L'hétérolinguisme chez Miano : à propos des littératures orales}

Maître d'École disait des choses, nous répétions. (...
) Nous nous donnions beaucoup de peine mais les
sons qui sortaient de nos bouches étaient
embrochés, les accents déformés, pimentés et
bâtonmanioqués. Notre français était mis à la page
au son du tam-tam, aux ricanements du balafon,
aux cris des griots.
(Calixte Beyala, 1994: 93-94)

3 Il n'y a pas une littérature orale, il existe des littératures orales qui s'expriment au gré des traditions culturelles qu'elles représentent. La fusion entre oralité et écriture est l'un des casse-tête de la théorie littéraire postcoloniale. Les difficultés à traduire les langues africaines, les grammaires des langues dites « orales », l'impossibilité de transmettre les rythmes, les assonances, les allitérations, les jeux de mots, les rimes, les dictons, les proverbes, le langage non verbal, les diverses figures et images symboliques complexifient l'appropriation de cet héritage infiniment riche d'enseignements et de connaissances, aussi bien chez les occidentaux que chez les 
africains « eux-mêmes » qui vivent désormais disloqués entre plusieurs modes et divers codes de représentation. ${ }^{5}$

4 Si les langues imprimées (cf. Barbier, 2008) ${ }^{6}$ tentent de restituer de façon spectaculaire les séquences du passé, non sans turpitudes ${ }^{7}$, à travers des documents écrits et des modes de muséification tous azimuts, les limites des langues non imprimées, autrement langues dites orales "par défaut", résident dans l'archivage des données, dans le bouche-àoreille, trop réducteur pour une distribution massive et une diffusion suprarégionale et pérenne. La parole représente ainsi un musée vivant et dynamique en soi qui, cependant, volera en éclats à la mort du détenteur des savoirs, lorsque la transmission n'a pas été effectivement assurée : ce qui arrive assez souvent.

En situation postcoloniale, les contradictions opérées dans la délimitation des champs théoriques passibles de connaissance - délimitation basée sur le principe de l'unité où il n'est admis que l'étude d'objets homogènes - sont inouïes. Ce principe est souvent appliqué spécialement aux littératures contenues dans le corpus ou canon national en expulsant de cet espace toutes les manifestations littéraires qui ne coïncident pas avec la norme, c'est-à-dire le discours dominant devenu classique, normal, standard, habituel, officiel ${ }^{8}$ , de rigueur suivant le critère de l'exclusion. Ce procédé réducteur est nu de son évidence. Le concept de littérature se rapporte exclusivement à la littérature "culte $»^{9}$, produite dans la langue héritée de la colonisation à travers les poèmes et livres de Victor Hugo, Molière, Balzac, Baudelaire, Montaigne, Rabelais, Montesquieu, Voltaire, Flaubert, etc., (et quelques "nègres-latins exceptionnels») où les rapports sociolectaux hybrides sont gommés et les textes, rédigés dans une curieuse langue artificielle, fictive, sans contagion, appelée langue pure, intolérante, distante, totalement externe au vécu du lecteur, pour solliciter en cette langue ce qu'elle a de plus orthodoxe (Chamoiseau, $1997: 274) .{ }^{10}$

En revanche, la littérature orale (produite dans son ensemble dans les langues " autochtones», minorisées) et la littérature populaire en français sont farouchement exclues de ce cadre. ${ }^{11}$ Le discours officiel recourt à leur expulsion partielle, totale ou définitive, en les renvoyant à un usage folklorique. En d'autres occasions, plus clémentes, le mécanisme est plus subtil et non moins insidieux: on situe ces littératures à un âge préhistorique et caverneux, comme si elles avaient cessé de se produire avec l'avènement de la colonisation. Dans le fond, d'une manière ou d'une autre, on retrouve là une manigance sinueuse qui leur applique une double négation, en ne leur reconnaissant tacitement aucune valeur littéraire ni aucune représentativité sociale, alors qu'objectivement il est impossible de nier la validité esthétique de ces dernières, tout comme il n'est même pas sensé ni logique de discutailler leur enracinement au sein des populations nationales dont elles sont issues, à moins de recourir au déni. Evidemment, il s'agit là d'une opération maladroitement idéologique (donc de légitimité) qui reproduit l'ordre politique et tente de justifier la hiérarchie des communautés linguistiques ${ }^{12}$ au sein d'un corpus national faussement monolithique, en marginalisant les littératures minorisées. ${ }^{13}$

7 La démarche consisterait donc à poser le problème en d'autres termes. Dans l'essentiel, l'équation pour une meilleure redistribution consisterait à redéfinir ou à déconstruire le concept d'unité qui, comme on l'a compris, est synonyme de partialité, en envisageant dès lors l'inclusion d'objets hétérogènes. Dans ce sens, en postcolonie le corpus et sa critique devra s'habituer (ou se réhabituer) à travailler, en consonance avec son matériel, sur des objets contradictoires, pluriels par essence, c'est-à-dire n'opposant pas de façon mécanique l'écriture à l'oralité, les identités hybrides étant le fruit de ces rencontres-là, 
hétérogènes. Une telle approche consisterait à travailler de ce fait sur des objets littéraires lesquels, dans leur composition, reflèteraient le caractère hétéroclite d'une littérature réellement nationale, située à la croisée de plusieurs systèmes sémiotiques. Ainsi, l'on réhabiliterait l'hybridation de cette littérature nationale-là. Le champ d'observation ici proposé serait considéré comme un espace d'expérimentation méthodologique, de peaufinage esthétique et d'approfondissement théorique. Car la critique littéraire post-nationale depuis ses fondements, avait alors considéré que ses connaissances ne pouvaient seulement s'apprécier que sur des corpus unitaires plus ou moins homogènes, probablement impulsés par les schémas adoptés par l'histoire des littératures nationales européennes, lesquelles avaient mis l'accent sur l'unité de leur matériel. Dans leur contexte, il s'agissait du processus historique qui le constituait, de sorte que le corpus coïncidât avec les limites de son système, s'assurant ainsi dans ses mécanismes de constitution une exclusivité unique et monolithique. Or, en postcolonie, en se l'appropriant dans un contexte hybride et disparate, il ne devrait y avoir de doute sur le caractère discrètement idéologique de l'opération. D'une part, le stratagème reproduisait et tentait de valider la hiérarchie et l'ordre politiques d'une société dominée. ${ }^{14}$ D'autre part, au niveau spécifique de la littérature, l'opération validait et intériorisait l'universalisme galvaudé du corpus colonial, dominant. Cependant, ce modèle ou canon admis et adopté, il trahissait l'échec des bourgeoisies postcoloniales ${ }^{15}$, incapables de formuler des projets politiques nationaux autonomes et suffisamment intégrateurs.

\section{Le conte chez Miano}

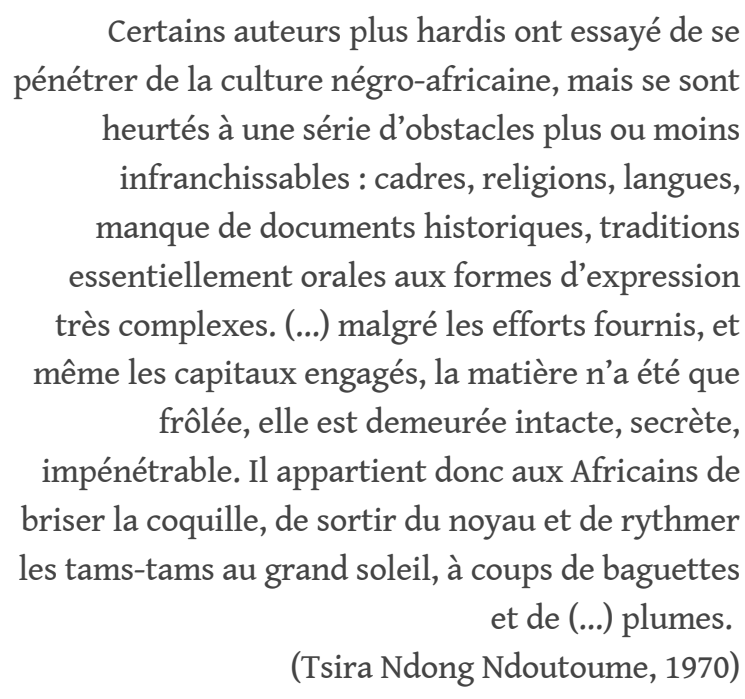

Généralement d'auteur anonyme, le conte est un patrimoine collectif, un outil didactique et interactif de la pédagogie « orale». L'art du conteur se nourrit de l'imaginaire d'une unité linguistique propre à une aire culturelle spécifique. La technique du conte réside dans les structures conventionnelles qui l'initient, l'accompagnent (refrains et chants génériques) et le dénouent. Le conte est essentiellement composé d'un récit (plus ou moins long) et d'une série de chants itératifs, allègres, relativement courts qui l'entrecoupent, en guise d'intermèdes ou d'entractes pour éviter la monotonie (d'un récitfleuve). Le chant permet de faire communier le conteur et le public. Rarement convié comme simple spectateur passif, afin de faire de lui un co-acteur/co-auteur, le public entonnera les chants sous la coordination de «l'artiste-pédagogue-conteur ». Puis, pour 
clore la séance, le narrateur-pédagogue résume la moralité de l'histoire racontée. Il en expose les principales idées et met fin au voyage imaginaire par une formule conventionnelle connue de tous, de la même façon qu'il l'a initié par une formule connexe. Au cours du récit, l'art oratoire du conteur est généralement accompagné par une gestuelle expressive presque théâtrale, le langage corporel y jouant un rôle déterminant pour maintenir le public en haleine. ${ }^{16}$

Nous allons voir comment Miano illustre des pans du conte dans Contours du jour qui vient (2006) où le conteur est symbolisé par une protagoniste du troisième âge, une grandmère, figure tutélaire et généalogique d'un musée vivant. Il n'est pas aléatoire non plus que pour assurer le relais de la transmission le public soit symboliquement représenté par la nouvelle génération dans une approche paritaire, une fille et un garçon, ses petitsfils :

Asseyez-vous là, mes enfants. Je vais vous raconter une histoire. (...) elle lance : Enguinguilayé! Nous répondons vivement: Ewésé! La réponse doit être aussi vive que l'appel du conteur, afin de témoigner de la qualité d'écoute de l'assistance. Nous sommes tout ouie (ibidem : 233).

10 À la suite de ce fragment, Miano prend le soin de mettre une note de bas de page pour expliquer le sens du terme «Enguinguilayé! » qui est une formule introductive que lance un conteur de l'ethnie douala pour s'assurer de l'attention du public. En retour, ce public lui répondra: «Ewésé»:

Enguinguilayé est un appel que lance le conteur, chez les Doualas du Cameroun. Il est répété par intervalles réguliers, pour vérifier que l'auditoire écoute bien. Le public ainsi interpellé répond: Ewésé. C'est un peu comme le Yé Krik (appel) Yé Krak (réponse) des Antillais. (ibidem : 233).

11 Par la suite, elle ajoute : "Cela fait partie des choses qui ne se traduisent pas » (ibidem: 233). Chez Léonora Miano - ainsi qu'on aura également l'occasion de l'observer chez Cristina García -, la note tente de sauver les apories qu'opère la double conscience linguistique de l'auteur. ${ }^{17}$ Ici, les productions hybrides ainsi que les représentations esthétiques s'inscrivent dans une dynamique dialogique où le composite, le transversal fait sien «le droit à l'opacité » de Glissant qui n'est pas le renfermement sur soi mais la trace dans l'autre langue de l'irréductible (1997 : 28-29). En sanctionnant que « [c]ela fait partie des choses qui ne se traduisent pas ", Miano soulève la problématique, en traductologie, des "essences intransférables", du cultural gap, du cultural turn, de l'incommensurabilité et de l'intraductibilité de codes culturels spécifiques. Sur ce point, doit-on retenir avec Sartre que « les forts en version n'existent pas. Cela tient à la nature du Verbe : on parle dans sa propre langue, on écrit en langue étrangère » (1964). Par la suite, Miano précise que « [i]ci, les contes ne servent pas à endormir, mais à éveiller. Ce sont des leçons de vie, destinées aux petits comme aux grands" (ibidem: 234). Nous retenons ainsi de la moralité de ce conte - et par ricochet du roman - que

[1]a colère est une illusion. Elle n'a rien à voir avec la force qu'elle simule mal. Ce que vous devez faire pour épouser les contours du jour qui vient, c'est vous souvenir de ce que vous êtes, le célébrer et l'inscrire dans la durée. Ce que vous êtes, ce n'est pas seulement ce qui s'est passé, mais ce que vous ferez (idem : 236). 


\title{
L'hétérolinguisme chez Cristina García : ¿Borrar el acento? ¿Pa'qué? ${ }^{18}$
}

\author{
(...) our tortured alphabets with the mysterious \\ tilde over the $n$ to make $\tilde{n}$, the $\ddot{u}$, the double \\ consonants $l l$ and $r r$ with their strong sounds. / \\ Cubanía: escribir con acento - en inglés. \\ (Santiago, 1994b : 31/Pérez Firmat, $2000: 105)$
}

De toute part, même dans les espaces et lieux les moins insoupçonnés, les Etats-Unis sont en train d'acquérir le « $\tilde{n}$ », un accent en espagnol. Il s'agit évidemment d'un passé commun sur lequel ont survécu et fleuri ces nombreuses cultures transfrontalières apparemment distantes et distinctes: les anciennes civilisations amérindiennes aujourd'hui "conquises", le catholicisme, la colonisation anglo-saxonne, espagnole, lusophone, italienne, française etc., et une grande partie des langues indigènes.

La frontière qui sépare les Etats-Unis de l'Amérique Latine est plus une imposition politique qu'une division géographique. C'est un câble avec une décharge électrique qui attire et repousse. Malgré tout, à la frontière les langues et les cultures se choquent, s'entremêlent, s'exploitent, se redéfinissent, cultivent sans cesse de nouveaux lexiques et produisent des identités hirsutes (Cristina García : 2006/2007). ${ }^{19}$ À la frontière, les langues et leurs identités se construisent des réalités alternatives - non sans frictions ni tensions inhérentes à la cohabitation -, car la frontière est un lieu fertile pour rêver. Rêver, voyager, pour qui est né à la frontière, constitue ainsi un second souffle. De par sa conscience de la circulation des mondes et de son expérience de plusieurs modes de vie, le transfrontalier, ici l'écrivain de la frontière, de l'entre-deux (ou plusieurs) a développé au détour de ces mouvements, une incalculable richesse du regard et de la sensibilité (Mbembe, ibidem).

\section{Spanglish, espanglés, oye cómo ladra la lengua de la frontera ${ }^{20}$}

Dans l'introduction à la version espagnole (1994b) de When I was Puerto Rican (1994a), introduction absente de la version originale anglaise, Esmeralda Santiago, une «jibara norteamericana ", écrivaine étasunienne d'origine portoricaine expliquait que la vie narrée dans son roman fut vécue en espagnol bien qu'initialement «transcrite » en anglais. Elle ajoute dans cette introduction qu'à plusieurs occasions, en écrivant, elle se surprenait en train de s'écouter parler en espagnol alors que ses doigts tapaient la même phrase en anglais. Un dilemme où des réalités transcrites en anglais étasunien parlaient de son enfance portoricaine alors que l'une ou l'autre langue ne pouvait tout traduire sans trahir la sémantique, les sens et les contextes. C'est pourquoi elle a prévu un glossaire à la fin du texte pour un public monolingue. Dans cet exercice, elle se retrouvait lovée dans une bulle langagière frustrante, bilingue, partagée entre l'espagnol et l'anglais. Elle reconnaît cependant que sa langue d'écriture, sa langue professionnelle et la langue qu'elle parle le plus est l'anglais, parce qu'elle vit aux Etats-Unis où la majeure partie des habitants est monolingue. Mais dans l'intimité profonde, les pensées qui habitent son intimité sont en espagnol. Ses rêves sont un mélange d'espagnol et d'anglais, souligne-t-elle. Cet exercice 
de traduction lui a fait prendre conscience que la langue qu'elle identifiait maladroitement à l'espagnol n'était en réalité que le spanglish, un anglais saupoudré d'espagnol [et vice versa] que parlent les hispanounidenses (les hybrides «latinos») lorsqu'ils croient parler l'une ou l'autre langue. Une langue-franche, dynamique, forgée par l'espagnol et l'anglais; une langue composite qui prend les expressions de part et d'autre en renouvelant l'étymologie des mots pour créer de nouveaux morphèmes, de nouveaux sens. Une langue-tampon dont la composition n'est pas le fruit d'une conjonction de «normes » apaisées et conventionnelles. Une langue de l'interposition née du chaos-monde, bâtie dans les marges, avec toutes sortes de matériaux qui par nature échappent à la patience de la règle. Le spanglish, une langue qui vit dans la précipitation, le besoin, l'angoisse et l'urgence de s'auto-définir :

Chicano Spanish is a border tongue which developed naturally. Change, evolución, enriquecimiento de palabras nuevas por invención o adopción have created variants of Chicano Spanish, un nuevo lenguaje. Un lenguaje que corresponde a un modo de vivir. Chicano Spanish is not incorrect, it is a living language.

Por a people who are neither Spanish nor live in a country in which Spanish is the first language; for a people who live in a country in which English is the reigning tongue but who are not Anglo; for a people who cannot entirely identify with either standard (formal, Castillian) Spanish nor standard English, (...) A language which they can connect their identity to, one capable of communicating the realities and values true to themselves - a language with terms that are neither español ni inglés, but both. (...) a patois, a forked tongue, a variation of two languages ( Anzaldúa, 1987: 55).

\section{La santería}

Dans son premier roman, García se sentait comme une étrangère en écrivant sur la santería ainsi qu'elle l'a déclaré au cours d'un entretien. Son contact avec la culture cubaine était alors le fruit de souvenirs évanescents puisqu'elle avait moins de trois ans lorsque ses parents et elle ont immigré aux Etats-Unis à la suite de la "Révolution Cubaine ». Parler de la santería fut donc un prétexte, une quête identitaire, pour aller à la rencontre de Cuba :
Me siento una extranjera al escribir sobre la santería. No la manejo y tal vez se hace evidente cuando he escrito sobre ella. En la actualidad conozco un poco más porque tengo una prima que se ha hecho santera. Cuando vine a Cuba en 1984 ella era una adolescente, pero, ahora, a través de ella he podido conocer a otras santeras, he asistido a ceremonias y me siento un poco más cercana a estas creencias. Para ser sincera, no pienso realmente en el público al abordar estos temas y tampoco si son exóticos o no. Lo que hago es que a través de los personajes me exploro a mí misma, pues son mis propias obsesiones, es una especie de cruz personal (Vitalina, 2002: 153).

16 Nous analyserons la «cubanisation" de l'anglais chez la romancière à travers les thématiques développées et les sujets abordés comme praxis d'une hybridité textuelle. Les effets de saturation descriptive - proches du surréalisme et du baroque - présentant les temples santeros sont une des clés de voûte de cette technique dont voici l'illustration :

Against the back wall, an ebony of Santa Bárbara, the Black Queen, presides. Apples and bananas sit in offering at her feet. Fragrant oblations crowd the shrines of the other saints and gods: toasted corn, pennies, and an aromatic cigar for Saint Lazarus, protector of paralytics; coconut and bitter kola for Obatalá, King of the White Cothe; roasted yams, palm wine, and a small sack for Oggún, patron of metals. In the front of the room, Elleguá, god of the crossroads (...) Four mulattas, 
wearing gingham skirts and aprons, kneel before the shrines, praying. One man, a pure blue-black Yoruban, stands mute in the center of the room, a starched cotton fez on his head (García, 1993: 13-14). espagnol dans un texte rédigé en anglais témoignent de la transversalité culturelle du narrateur. Le champ lexical santero - expressément reproduit en espagnol et en langues africaines (héritage de l'esclavage) -, décrit des réalités spécifiques, cubaines au moyen d'idiotismes, c'est-à-dire de prêt lexical que l'anglais ne peut pas traduire. Dans les fragments de texte, nous avons souligné en gras l'étrangeté de ces réalités incommensurables, opaques :

The oddu, the official santería prediction for this year, is mixed. (...) In the second week of January, Felicia visits a santero known for his grace and power in reading the divining shells. Through the mouths of the cowries the gods speak to him in clear, unambiguous voices. The santero (...) begins to pray in Yoruba, asking for the blessings of the orishas, whom he honors one by one. (ibidem : 147-148).

Pour échapper au classique glossaire en fin de texte ou pour éviter les intempestives notes en bas de page, assez impertinentes et incommodes pour la lecture, García opte pour une traduction en simultané, comme :

I told her that at my house we had many shells, that they told the future and were the special favorites of Yemayá, goddess of the seas. (...) Felicia's parents were afraid of my father. He was a babalawo, a high priest of santería (...) His godchildren came from many miles on his saint's day, and brought him kola nuts and black hens. (...) Once she saw my father use the obi, the divining coconut, to answer the questions of a godchild who had come to consult him. I remember the pattern of rinds fell in ellife, two white sides and two brown, a definite yes (idem: 183-184-246).

Dans un procédé analogue, nous avons un fragment similaire dans lequel, pour échapper au glossaire, la narratrice choisit, dans un autre roman, de recourir à nouveau à une traduction simultanée, sans oublier au passage les récurrentes allusions aux concepts cubains reproduits dans les langues d'origine :

"The shells never lie. Through their mouths the orishas disclose their purpose." (...) The pattern falls in ofún, where the curse was born. This is the principal ruling. Then he throws the shells twice more. The message doesn't waver: oddi, where the grave was first dug. (...) The santero continues to pray. Her luck is not good, he says. (...) Before long, La Madrina initiated Felicia into the elekes and gave her the necklaces of the saints that would protect her from evil. (...) Sixteen days before the asiento, Felicia went to live with La Madrina, who had procured seven white dresses for her, seven sets of underwear and nightclothes, seven sets of bedding, seven towels, large and small, and other special items, all white. (...) On the morning or her initiation, sixteen santeras tore Felicia's clothes to shreds until she stood naked, then they bathed her in river water, rubbing her with soap wrapped in vegetable fibers until her skin glowed. The women dressed Felicia in a fresh white gown and combed and braided her hair, treating her like a newborn child.(...) La Madrina slipped the sacred necklace of Obatalá around Felicia's neck.(..) After many more rituals and final bath in the omiero, the santeras led Felicia to Obatala's throne. The diviner of shells shaved her head as everyone chanted in the language of the Yoruba (1993: 186). Felicia had left a note with Herminia saying she wanted to be buried as a santera, and Celia could not refuse her daughter's last request. In the mortuary, her friends from the casa de santo dressed Felicia in her initiation gown, her crown, and her necklaces (1997: 109- 214).

20

L'écriture de l'étrangeté ou l'esthétique de l'intranquillité recourt parfois à d'autres stratagèmes. Hormis l'écriture en italique de mots étrangers et les traductions en 
simultané, dans le fragment suivant nous avons droit aux parenthèses pour traduire l'altérité.

The owner of the shop is an elderly man who wears a white tunic and cotton fez. For a young woman with cropped hair, he prescribes a statuette of La Virgen de la Caridad del cobre, a yellow candle, and five special oils: amor (love), sígueme (follow me), yo puedo y tú no (I can and you can't), ven conmigo (come with me), and dominante (dominant), (1993: 199).

21 Nous avons énuméré tout au long de ce travail les différents modes de combinaison de l'hybridité dans les textes issus des identités-frontière(s): de l'écriture en italique aux parenthèses, en passant par les notes de bas de page et les traductions simultanées. Toutefois, nous n'avons pas analysé le cas des glossaires en fin de texte et l'hybridation des structures linguistiques internes. Il est donc important de préciser que, comme toute étude théorique, rien de ce qui est affirmé dans ce travail n'est définitif, davantage dans un domaine aussi prolifique, dynamique et polymorphe que l'identité, la frontière et les langues vivantes.

Pour clore, il nous plaît de partager une anecdote rapportée par l'écrivain cubain Alejo Carpentier (1987: 121) à propos de La vorágine du colombien José Eustasio Rivera. Il explique que lorsque son confrère colombien a publié son texte en 1924, à la fin de l'œuvre, il offrait un glossaire de près de deux-cent-vingt américanismes, à l'époque, inconnus de la Real Academia Española. En utilisant ces mots nouveaux pour l'espagnol de la Péninsule Ibérique, cela constituait à n'en point douter une évidente limitation et une grosse audace. Écrire de cette façon, avec des mots jugés " exotiques ", " étranges ", "barbares ", " grossiers ", hartos localistas, était audacieux certes, néanmoins il ne passait pas par l'angoisse et l'impuissance de Hernán Cortés qui, dans ces correspondances à Carlos V se plaignait, en son temps, de ne pouvoir nommer ni décrire à suffisance des réalités amérindiennes qui n'avaient pas de parangon en espagnol. En 1927, avec jÉcueYamba-O !, roman negrista, Carpentier suivra les traces de son confrère, en optant pour un glossaire de cubanismes inconnus des lecteurs hispano-américains et espagnols. Carpentier observera par la suite qu'en relisant $L a$ vorágine des années plus tard, une quarantaine de ses «barbarismes» d'alors avaient été ajoutés aux dictionnaires espagnols, en devenant ainsi un vocabulaire usuel, loin des « localismes incorrects » et de l'exotisme qu'on leur trouvait. Il ajoute qu'à partir de la décade de 1930-1940, la peur des américanismes s'est estompée parce qu'il fallait bien nommer des choses par leur nom...

Analyser la frontière c'est faire irruption au milieu d'une phrase, éclater une norme standardisée en combinant des éléments disparates au point de donner l'impression d'un confusio linguarum. C'est pourquoi ce travail semble trop en demander au lecteur monolingue de par son contenu et son style, mais le chemin des écritures-frontière(s) frise inévitablement un esperanto poétique.

Comment écrire hybride lorsqu'on est situé à l'embouchure de plusieurs langues, c'est-àdire dans l'orbite de la transversalité ? Comment $d$ /écrire la frontière dans une fraicheur 
lexicale où les langues feraient du bouche-à-bouche, sans avoir besoin de les juxtaposer ni de les superposer?

Chez nos écrivaines, les modifications évidentes au niveau du lexique que permet une langue dominante comme le français ou l'anglais se situent essentiellement aux trois niveaux suivants :

- l'incorporation de mots étrangers qui expriment dans l'autre langue ou dans la langue de l'autre la spécificité culturelle que l'on veut nommer, et on parlera alors de prêt lexical ou d'adoption;

- la recréation ou réélaboration d'un concept à partir des outils linguistiques de la langueréceptrice, on l'appellera " calque sémantique » ou tout simplement " traduction »;

- l'appropriation d'un concept de l'autre langue (ou de la langue de l'autre) par la réorientation sémantique d'un vocable préexistant, à défaut de trouver mieux on dira resémantisation (Martin Lienhard, 2003). ${ }^{21}$

On dira donc, pour paraphraser Lise Gauvin, qu'à cause de sa situation particulière créée par des publics immédiats ou éloignés, séparés eux par des historicités et des acquis culturels et langagiers différents, l'écrivain hybride est condamné à penser/panser la langue. Car la situation de diglossie sociale dans laquelle il se trouve le plus souvent immergé l'oblige à recourir à une "stratégie du détour". Stratégie multiforme qui va de la transgression à l'intégration en passant par la traduction, sans compter les tentatives de normalisation d'un certain parler vernaculaire ou encore la mise en place de systèmes astucieux de cohabitations de langues ou de niveaux de langues, qu'on désigne généralement sous le nom de plurilinguisme ou d'hétérolinguisme textuel (Gauvin, 2000 : 6-8).

Plus que des réponses, notre travail soulève ici des questionnements profonds dans un élan de renouvellement. Plus que l'hybridité, on a soulevé ici la problématique des langues en situation diglossique dans un contexte de négociation de pouvoir où les langues minorisées résonnent en sourdine, comme un concert d'interférences disjointes, dans la langue dominante ou officielle pour mieux la conquérir. Car, il faut bien le reconnaitre, certaines langues assises sur un système morphosyntaxique "stable » ne tolèrent qu'assez rarement de profondes modifications. C'est pourquoi, dans certains contextes d'écriture comme l'hétérolinguisme, l'introduction sporadique de sociolectes ne s'observe, de façon astucieuse et sibylline, que dans le recours lexical et dans les contextes circonscrits comme les dialogues où les personnages peuvent parler à leur guise.

L'hybridité n'aura plus besoin de traduire lorsque les langues ne seront plus hiérarchisées, que l'on n'éprouvera plus le besoin de mettre la note en bas de page, ou en fin de volume; bref, lorsque le donné du monde sera là comme les autres, sans explications. Car il en va des civilisations comme des langues : stagnantes, elles doivent leurs rebonds à leurs rebords, à leurs changements de portage quand elles en rencontrent une autre, exotique jusqu'alors (Debray, 2010: 81-82). Il faudra donc impulser des bouleversements, des avancées, de l'audace, des chocs, des harmonies qui soient intéressantes à tracer dans l'effort des littératures du monde. 


\section{BIBLIOGRAPHIE}

ANZALDÚA, Gloria (1987). Borderlands La Frontera, The New Mestiza. San Francisco: Aunt Lute Books cop.

ASHCROFT, Bill, GRIFITH, Gareth et TIFFIN, Helen (2012). L'Empire vous répond: Théorie et pratique des littératures postcoloniales. Traduction de Jean-Yves Serra et Marine Mathieu-Job. Bordeaux : Presses Universitaires de Bordeaux.

BARBIER, Frédéric (2008). Les langues imprimées. Paris : Librairie Droz.

BHABHA, Homi K. (2007). Les lieux de la culture : Une théorie postcoloniale. Paris : Payot.

BEVERLY, John (2004). Subalternidad y Representación : debates en teoría cultural. Madrid :

Iberoamericana.

BEYALA, Calixte (1994). Assèze l'Africaine. Paris : Albin Michel.

BOURDIEU, Pierre (2001). Langage et pouvoir symbolique. Paris : Fayard.

CALVET, Louis-Jean (1974, rééd. 2002). Linguistique et colonialisme. Petit traité de glottophagie. Paris : Petite Bibliothèque Payot.

CARPENTIER, Alejo (1987). Tientos, diferencias y otros ensayos. Madrid : Plaza y Janés Editores.

CHAMOISEAU, Patrick (1997). Écrire en pays dominé. Paris : Gallimard.

CÉSAIRE, Aimé (1955, rééd. 1995). Discours sur le colonialisme. Paris : Présence Africaine.

CONDÉ, Maryse (1978). La civilisation du bossale. Réflexions sur la littérature orale de la Guadeloupe et de la Martinique. Paris : L'Harmattan.

CORCUERA IBÁNEZ, Mario (2009). Tradition et littérature orale en Afrique noire. Parole et réalité, préface de Léopold Sédar Senghor, tr. Alicia Bermoken. Paris : L'Harmattan.

CORNEJO POLAR, Antonio (1997). Mestizaje e hibridez: los riesgos de las metáforas. La Paz : Universidad Mayor de San Andrés.

CORNEJO POLAR, Antonio (1982). Sobre literatura y crítica latinoamericanas. Caracas : Universidad Central de Venezuela.

DEBRAY, Régis (2010). Éloge des frontières. Paris : Gallimard.

DORRA, Raúl (1997). Entre la voz y la letra. Universidad de Puebla-México : Plaza y Valdés Editores.

DERRIDA, Jacques (1971). De la gramatología. Buenos Aires : Siglo XXI.

ENO BELINGA, S.-M. (1978a). Comprendre la littérature orale africaine, les classiques africains. Issy les Moulineaux : Ed. Saint-Paul.

ENO BELINGA, S-M. et MINYONO NKodo, M.-F. (1978b). Poésies orales, collection les grands textes africains. Issy-les-Moulineaux : Saint-Paul.

FANON, Frantz (1961, rééd. 2002). Les damnés de la terre. Paris : La Découverte/Poche.

FANON, Frantz (1959, rééd. 2006). Pour la révolution africaine. Écrits politiques. Paris : La Découverte / Poche. 
FLEISCHER, Alain (2005). L'accent, une langue fantôme. Paris : Seuil.

GARCÍA CANCLINI, Nestor (1990, rééd. 2001). Sobre Culturas híbridas. Estrategias para entrar y salir de la modernidad. Barcelona : Paidos

GARCíA, Cristina (2007). A handbook to luck. New York: Vintage books.

GARCíA, Cristina (2006). Bordering fires. (The vintage book of contemporary mexican and chicano/a literature, edited and with an Introduction by Cristina García). New York: Vintage books.

GARCíA, Cristina (1993). Dreaming in Cuban. New York: Ballantine books.

GARCÍA, Cristina (1997). The Agüero sisters. Nueva York : Vintage español books.

GAUVIN, Lise (2000). Langagement, l'écrivain et la langue au Québec. Montréal : Boréal.

GLISSANT, Édouard (2002). Le Discours antillais. Paris : Gallimard.

HALL, Stuart (2008). Identités et cultures. Politiques des Cultural Studies. Edition augmentée. Paris :

Éditions Amsterdam.

KRISTEVA, Julia (1998, réédition 2007). Étrangers à nous-mêmes. Paris : Gallimard.

LIENHARD, Martin (2003). La voz y su huella. México : Ediciones Casa Juan Pablos (Universidad de Ciencias y Artes de Chiapas).

MATO, Daniel (1990). El arte de narrar y la noción de la literatura oral. Caracas : U.C.V. - CDCH.

MBEMBÉ, Achille : http://www.africultures.com/php/index.php?nav=article\&no=4248 (consulté le 09 juin 2015)

MEMMI, Alfred (1985). Portrait du colonisé (précédé de Portrait du colonisateur et d'une préface de Jean-Paul Sartre), Agence de coopération culturelle et technique, Francopoche. Paris : Gallimard. MIANO, Léonora (2006). Contours du jour qui vient. Paris : Plon.

MIANO, Léonora (2008). Afropean Soul et autres nouvelles. Paris : Flammarion.

MIANO, Léonora (2012). Habiter la frontière. Paris : L'Arche Editeur.

ONG, Walter J. (1987). Oralidad y escritura. México : F.C.E.

PÉREZ fIRMAT, Gustavo (2000). Cincuenta lecciones de exilio y desexilio. Miami : Ediciones Universal.

RAMA, Ángel (1984, rééd. 1998). La ciudad letrada. Montevideo : Arca.

SAIID, Edward (2000). Culture et impérialisme. Paris : Fayard/Le Monde diplomatique.

SAïD, Edward W. (1980). L'Orientalisme, L'Orient créé par l'Occident, traduit de l'américain par Catherine Malam. Paris : Seuil.

SANTIAGO, Esmeralda (1994b). Cuando era puertorriqueña. Nueva York : Vintage Español.

SANTIAGO, Esmeralda (1994a). When I was Puerto Rican. New York: Vintage Book.

SARTRE, Jean Paul (1964). Les mots. Paris : Gallimard.

SPIVAK, Gayatri Chakravorty (2009). En d'autres mondes, en d'autres mots. Essai de politique culturelle. Paris : Payot.

SPIVAK, Gayatri Chakravorty (2009). Les subalternes peuvent-elles parler? Traduit de l'anglais par Jérôme Vidal. Paris : Éditions Amsterdam. 
TORRES, María de los Ángeles (1994). "Beyond the Rupture": Reconciling with Our Enemies, Reconciling with Ourselves", Michigan Quarterly Review XXXIII.

TRILLES, HENRI (2002). Contes et légendes fang du Gabon. Paris : Karthala.

TSIRA NDONG, Ndoutoume (1970, rééd. 1983). Le Mvett épopée fang tome premier (paroles et traditions). Paris : Présence Africaine.

TSIRA NDONG, Ndoutoume (1993). Le Mvett, L'homme, la mort et l'immortalité. Paris : L'Harmattan.

VITALINA, Alfonso (2002). Ellas hablan de la isla. La Habana : Ediciones Unión.

http://www.lanouvelletribune.info/benin/culture/12951-polemique-dans-le-monde-scolaire-

beninois-autour-de-verre-casse-d-alain-mabanckou, consulté le 08 juin 2015).

WA THIONG'O, Ngugi (2011). Décoloniser l'esprit. Traduit de l'anglais par Sylvain Prudhomme. Paris : La fabrique.

zUMTHOR, Paul (1989). La letra y la voz de la "literatura" medieval. Cátedra : Madrid.

\section{NOTES}

1. Le chaos-monde glissantien enfante " le Divers, qui n'est pas le chaotique ni le stérile, [car il] signifie l'effort de l'esprit humain vers une relation transversale, sans transcendance universaliste. Le Divers a besoin de la présence des peuples, non plus comme objet à sublimer, mais comme projet à mettre en relation." (Glissant, $2002: 327)$

2. Paru pour la première fois en 1974, en pleine "décolonisation », Linguistique et colonialisme... analyse les rapports entre les langues et le politique, aussi bien entre des langues différentes (anglais par rapport français par exemple) qu'à l'intérieur d'une même unité linguistique (variantes dialectales et régionales). En mêlant histoire, géographie, intérêts politiques, économiques, religieux et scientifiques, Louis-Jean Calvet explique les divers mécanismes de domination exercée sur un peuple ou une communauté à travers «la glottophagie». Par l'entremise de l'édifice colonial rendu possible par des fondements scientifiques arbitraires pour justifier ou légitimer la hiérarchisation des langues, Calvet explique comment une langue en «mange » une autre.

3. Tout au long de ce travail, nous utiliserons le terme " minorisé » ou " minoré » en lieu et place de « minoritaire ", « minorité » parce qu'il est question ici d'un rapport de domination politique (ou du fruit d'une construction sociale) et non d'une infériorité numérique.

4. L'une des particularités de l'hybridité réside dans l'instabilité lexicale de la réalité composite que l'on veut traduire ou nommer. Le spanglish admet trois orthographies : espanglés (écrit en espagnol), spanglish (nom du concept en anglais), espanglish (prononciation espagnolisée du terme anglais).

5. Héritée de l'époque coloniale, et aujourd'hui intériorisée en situation postcoloniale, une relation binaire fétichisée, cachant une erreur monumentale, a été maintenue (et continue de l'être) en laissant croire que la langue coloniale, devenue langue officielle, c'est-à-dire la langue $\mathrm{de} / \mathrm{du}$ pouvoir, serait l'unique langue passible de connaissances. Or, dans les langues "autochtones" vivant en situation diglossique, il existe au sein des littératures qu'elles hébergent des niveaux de langue différents tout comme dans les langues imprimées. Il n'y aurait donc pas d'un côté des langues «ignorantes» et de l'autre des langues «savantes ». La langue comme véhicule de communication est un fait tandis que la langue comme domaine de la rhétorique "savante» est tout autre. Il faut au-delà de la langue comme vecteur de communication, avoir les outils grammaticaux indispensables pour accéder à toute littérature culte. 
6. L'invention de l'imprimerie permet aujourd'hui de constater «l'hégémonie » des langues écrites sur les langues « orales ».

7. «L'Afrique n'existe qu'à partir du texte qui l'a construite comme la fiction de l'autre. C'est à ce texte que l'on accorde ensuite une puissance structurante, au point où le soi qui prétend parler d'une voix authentiquement sienne encourt toujours le risque de ne jamais s'exprimer qu'à partir d'un discours préconstitué, qui masque le sien propre, le censure ou l'oblige à l'imitation. L'Afrique n'existe qu'à partir d'une bibliothèque coloniale qui s'immisce et s'insinue partout, $\mathrm{y}$ compris dans le discours qui prétend la réfuter, au point que, en matière d'identité et de tradition africaine, il est désormais impossible de distinguer l'original de la copie, voire de son simulacre » (http://www.africultures.com/php/index.php?nav=article\&no=4248). Cette assertion de Achille Mbembé ravive le débat sur l'Afrique qui n'existerait que comme un concept à partir du contexte, du prétexte voire du précepte qui l'a construite comme la fiction et la périphérie de l'autre. Cette périphérie n'existerait donc et ne prendrait sens qu'en référence au curseur de la norme dominante laquelle, à son tour, ignore que la binarité centre/périphérie qu'elle prône en sa faveur n'est qu'une forme d'ethnicité voilée de la dictature dominante.

8. Il y a des adjectifs apparemment anodins mais sibyllins qui cachent un discours de persuasion tapis dans les décombres de "l'autorité » et de la «légitimité » c'est-à-dire d'un pouvoir qui se veut dominant (parce que les combines de l'Histoire unilatérale l'ont autoproclamé dans l'absolu). Je me méfie habituellement des épithètes "pur», "normal», "authentique», « officiel », « classique », « correct », « naturel », «standard», «traditionnel », « habituel » associés pour la plupart à ce qui se « doit ». Là où ils apparaissent avec insistance, persistance et recrudescence sans indulgence, il y a invalidation de la diversité et inhibition des hybridations.

9. En 2012, la polémique soulevée au Bénin autour de Verre Cassé d'Alain Mabanckou, inscrit au programme scolaire 2012-2013 est une illustration frappante. Au mois d'octobre, le ministère des enseignements secondaires et techniques publiait la liste des ouvrages inscrits au programme scolaire. En classe de Terminale, Les Bouts de bois de Dieu d'Ousmane Sembène a été remplacé par Verre Cassé d'Alain Mabanckou. Deux mois après, les avis, dans le monde de l'éducation, divergeaient et prenaient l'allure d'une «guéguerre de tranchées». Deux tendances s'affrontaient. Les «normalistes » et les «transgressionnistes ». Pour les «normalistes », il fallait que les élèves eussent fini de maîtriser les normes de l'écriture «classique », de la narration «traditionnelle » avant de se risquer, à des échelons plus élevés, à la transgression. De l'autre côté, les arguments des «transgressionnistes » : certes, avancent-ils, le roman d'Alain Mabanckou est une œuvre surprenante qui déconstruit le mécanisme de la narration traditionnelle, mais elle s'inscrit parfaitement dans les nouvelles tendances de la littérature africaine. Et ces nouvelles tendances sont faites d'audaces, d'approches plus personnelles de l'écriture, de décalages stylistiques, une appropriation de la langue en adéquation avec les thèmes, plus actuels, de la société. (http://www.lanouvelletribune.info/benin/culture/12951-polemique-dans-le-mondescolaire-beninois-autour-de-verre-casse-d-alain-mabanckou, consulté le 08 juin 2015).

10. Chamoiseau (1997: 274) soutient que «la langue dominante quand elle est apprise comme extérieure à soi, se conserve à distance: on la manie en demandeur; voulant la conquérir, on sollicite ce qu'elle a d'orthodoxe».

11. Dans Les subalternes peuvent-elles parler?(2009), Gayatri Spivak soulève la question de la confiscation de la parole par les élites bourgeoises postcoloniales. La plupart ayant été éduquées à la trique européanocentriste reproduisent (in)consciemment les modèles « ailleurisants ». Elle interroge donc la symbolique des rapports de domination dont le plus saillant réside dans la réappropriation du discours par les subalternes non plus comme objets mais comme sujets du discours. John Beverley étudie aussi la même question dans Subalternidad y Representación: debates en teoría cultural.

12. Dans Langage et pouvoir symbolique, Bourdieu analyse les relations et stratégies de pouvoir à travers les échanges linguistiques exprimés à des degrés différents: variations d'accents, 
d'intonation et de vocabulaire qui reflètent les différentes positions dans la hiérarchie sociale. L'on comprend alors que « les individus parlent avec différents degrés d'autorité, que le poids des mots dépend de celui qui les énonce et de la façon dont ils sont formulés, et qu'ainsi certaines paroles, prononcées dans certaines circonstances, ont une force et une conviction qu'elles n'auraient pas autrement ». Ainsi, « les mots deviennent autant d'instruments de coercition et de contrainte, d'abus et d'intimidation, de signes de politesse, de condescendance et de mépris ». Le concept majeur qu'emploie Bourdieu lorsqu'il développe son approche est celui d'habitus, terme très ancien dont on sait les origines aristotéliciennes et scolastiques, mais dont Bourdieu fait un usage tout à fait distinct et spécifique. L'habitus désigne un ensemble de dispositions qui portent les agents à agir et à réagir d'une certaine manière. Les dispositions engendrent des pratiques, des perceptions et des comportements coordonnées et régis par aucune "règle". Les dispositions qui constituent les habitus sont inculquées, structurées, durables; elles sont également génératives et transposables. (...) En tant qu'ensemble de dispositions durablement installées, l'habitus tend également à engendrer des pratiques et des perceptions, à produire des œuvres et des appréciations, accordées avec les conditions d'existence dont l'habitus est lui-même le produit (ibidem : 7-24-25).

13. Revue critique sur la question en Amérique Latine: Antonio Cornejo Polar (1982) fait une exégèse de l'historiographie littéraire pour expliquer l'exclusion des épistémologies "périphérisées » à travers les mécanismes de domination légués des archétypes coloniaux. Cette démarche est proche de l'épistémè foucaldienne (Les mots et les choses) où la domination ne se limite pas à une idéologie théorique mais s'étend jusqu'à l'édifice qui supporte les productions intellectuelles et culturelles propres à un contexte, lequel reproduit dans le discours et l'action le clivage centre/périphéries en réduisant au statut d'objet tout ce qui est considéré étranger. Cornejo Polar expose que les corpus littéraires latino-américains, ayant gommé toute transversalité permettant d'inclure les littératures précolombiennes, travaillent de ce fait sur des corpus erronés car ceux-ci sont unilatéralement conditionnés par les canons hérités des littératures coloniales. Ángel Rama (1984) explique ainsi l'obsolescence des systèmes précoloniaux en établissant les rapports de causalité entre l'écriture (imprimée) et les structures de pouvoir. Martin Lienhard (1989), lui, considère la «fétichisation de l'écriture » et ses effets immédiats sur les systèmes de communication verbale comme un processus d'acculturation ethnocidaire au détriment des typologies de l'oralité amérindiennes. García Canclini quant à lui propose l'hétérogénéité comme refondation épistémique d'une critique littéraire latinoaméricaine sans exclusive: une sorte de poétique de la Relation glissantienne ou de transculturación actualisée du cubain Fernando Ortiz ou encore du concept de la diversalité chère à Raphaël Confiant.

14. Patrick Chamoiseau (1997) a décrit la violence névrotique d'une «écriture dominée » dans un contexte de dépersonnalisation absolue (Frantz Fanon, 1961) où l'imaginaire c'est-à-dire «le moded'être, le mode-de-penser, le mode-de-se-penser, le mode-de-penser-sa-pensée, s'abreuve du matin jusqu'aux rêves, à des images, des pensées, des valeurs qui ne sont pas siennes ». Pour une meilleure analyse, on peut aussi relire l'essai pamphlétaire anticolonialiste d'Aimé Césaire (1955) et revoir « la catalepsie sociale et historique du colonisé » d'Albert Memmi (1985) afin de décoloniser l'esprit (Wa Thiong'o, Ngugi 2011). De plus, Edward Saïd (1980, 2000), Spivak (2009) et Stuart Hall (2008) analysent, à divers degrés, l'entreprise de domination "des anciens empires» en proposant dès lors des schèmes alternatifs de représentations politiques ainsi que de possibles stratégies de reconnaissance.

15. L'épithète "postcolonial » utilisé tout au long de ce travail est à comprendre selon la perspective de Bill Ashcroft (2012) qui le définit non comme une rupture chronologique ni dichotomique (post-colonial) mais plutôt comme «toute culture affectée par le processus impérial depuis le moment de la colonisation jusqu'à nos jours ». Dans ce domaine, Homi K. Bhabha (2007) souligne que le postcolonialisme « témoigne des forces inégales et inégalitaires de 
représentation culturelle qui sont à l'œuvre dans la contestation de l'autorité politique et sociale au sein de l'ordre mondial moderne ».

16. Tsira Ndong Ndoutoume (1970, 1993), Eno Belinga (1978a et b), Maryse Condé (1978), Henri Trilles (2002), Corcuera Ibánez (2009), Jacques Derrida (1971), Walter J. Ong (1987), Paul Zumthor (1989), Martin Lienhard (2003), Daniel Mato (1990), Raúl Dorra (1997) et bien d'autres exposent de façon remarquable les pratiques épistémiques et discursives des productions orales.

17. Miano dit: " La langue française, telle que je l'écris, est mâtinée d'africanismes, d'anglicismes, de créolismes. Tout cela compose mon univers. Il ne s'agit pas seulement d'emprunts lexicaux, mais aussi d'images propres aux cultures africaines et créoles. Les anglicismes sont, le plus souvent, des transpositions directes, des traductions littérales, qui donnent des choses qui «ne disent pas» en français. (...) Le Cameroun d'où je suis originaire a deux langues officielles, le français et l'anglais. Même dans la partie francophone du pays, où je suis née, on parle un pidgin english, et un franglais bien connus de ceux qui ont visité le Cameroun. Pour moi, il est donc normal d'imbriquer ces langues l'une dans l'autre. » (2008: 95)

18. Tr. : Effacer l'accent? À quoi bon?

19. Très proche de la perspective glissantienne, dans Habiter la frontière (2012: 25), Léonora Miano expose tout comme Cristina García que la frontière, telle qu'elle la définit et l'habite, est «l'endroit où les mondes se touchent, inlassablement. C'est le lieu de l'oscillation constante... C'est là où les langues se mêlent (...) s'imprégnant naturellement les unes les autres, pour produire, sur la page blanche, la représentation d'un univers composite, hybride. La frontière évoque la relation. Elle dit que les peuples se sont rencontrés, quelquefois dans la violence, la haine, le mépris, et qu'en dépit de cela, ils ont enfanté du sens. (...) Elle rappelle, à ceux qui croient en la fixité des choses, des identités notamment, que non seulement la plante ne se réduit pas à ses racines, mais que ces dernières peuvent être remportées, s'épanouir dans un nouveau sol. »

20. Ecoute donc comment « aboie » (résonne) la langue de la frontière!

21. Patrick Chamoiseau pense que «la communication entre les peuples, la communication culturelle, ne passe pas par l'évidence. Ce n'est pas parce que je vais traduire tous les mots en créole que je vais entrer plus en communication avec qui que ce soit. Je crois à la communication qui se fonde sur des choses beaucoup plus mystérieuses et le jeu que je fais dans les traductions, c'est un petit jeu personnel qui n'entre pas dans une volonté d'éclaircir quoi que ce soit. Une note peut me permettre de faire un petit trait d'humour, de renverser la note habituelle plutôt que de faire une explication pour un Français ou un Martiniquais. Je joue une petite dérision de tous les glossaires qui généralement accompagnent les écritures et les littératures dites particulières. Je déjoue aussi le vieux schéma occidental. Le colonialiste a envie que l'autre lui ressemble, que l'autre soit transparent. À la limite, il préfère que l'autre n'existe pas du moment que sa transparence soit assurée. Le colonialiste vide toutes les zones intérieures de l'autre. L'autre est complètement édulcoré, éclairci, de façon à ce qu'on puisse voir à travers lui, et même se voir soi-même. C'est un dialogue qui n'en est pas un. Lorsqu'on se met à faire des glossaires et à expliquer, on entre dans ce schéma. Il n'y a pas de communication culturelle entre les peuples. (...) Je crois que, désormais, on peut jouer avec la note, les traductions, les parenthèses, et les voir comme un jeu littéraire, un jeu musical, un jeu poétique, et non comme un processus de clarification de texte» (Gauvin, 2000 : 43-44). Il rejoint le «droit à l'opacité » de Glissant qui signifie accepter l'autre avec ce qu'il y a d'irréductible en lui. 


\section{RÉSUMÉS}

Notre analyse est une étude transversale focalisée sur l'hétérolinguisme comme faits et effets de langue chez les écrivaines Léonora Miano et Cristina García. Nous voulons souligner les apories observées dans les littératures issues de l'entre-deux où la traduction joue un rôle primordial. Chez Miano la francophone, on étudiera les notions de l'oralité africaine à travers le conte tandis que chez la cubano-américaine d'expression anglaise Cristina García, nous parlerons du spanglish (ou espanglish ou encore espanglés) avec pour point nodal la santería cubaine. Le propos ici vise à mettre en exergue la littérature produite par les minorités vivant dans la diaspora au cœur de notre réflexion. Notre approche théorique sera pluridisciplinaire parce qu'un texte hybride, c'est-à-dire hétérogène donc à ancestralités multiples, requiert un regard pluriel pour mieux apprécier la polysémie de l'âme métisse des identités-frontières.

Our analysis is a cross-sectional study with a special focus on heterolinguism as language facts and effects in Leonora Miano and Cristina García's works. We want to highlight the aporias that are observable in hybrid (or in-between) literatures; where translation plays a pivotal role. In Miano's Francophone work, we will study the notions of African orality through storytelling. As for the Cuban American and English speaker Cristina García, we will discuss on Spanglish (or Espanglish or Espanglés) driving our analysis from the Cuban Santería. The subject here aims at highlighting this literature produced by minorities, with a specific focus on two main authors: Léonora Miano (from African European origins) and Cristina García (a Cuban American). In a time of heterotopic references, our theoretical approach will be multidisciplinary; for a fragmented or hybrid text (a heterogeneous text with multiple ancestralities) requires a plural look to better appreciate the polysemy of the mixed-identity soul within frontiers authors.

\section{INDEX}

Keywords : aporias, paratext, translation, heterolinguism, Cuban-American literature, French literature

Mots-clés : apories, paratexte, traductologie, hétérolinguisme, littérature cubano américaine, littérature française

\section{AUTEUR}

FERDULIS ZITA ODOME ANGONE

Université Cheikh Anta Diop/Dakar

Odome_zita[at]yahoo.fr 